\title{
Arbeits(zeit)schutz nach Art der BRD - am Beispiel der COVID-19-Arbeitszeitverordnung
}

\author{
Friedhelm Nachreiner ${ }^{1}$ \\ Angenommen: 23. April 2021 / Online publiziert: 17. Mai 2021 \\ ๑) Springer-Verlag GmbH Deutschland, ein Teil von Springer Nature 2021
}

\section{Zusammenfassung}

Das BMAS hat gemeinsam mit dem BMG im April 2020 für einen begrenzten Zeitraum die COVID-19-ArbZV erlassen, um die Patientenversorgung und die der Allgemeinbevölkerung sicherzustellen. Die darin genehmigten Abweichungen vom ArbZG stehen im scharfen Gegensatz zu den vorliegenden arbeitswissenschaftlichen Erkenntnissen zur Arbeitszeitgestaltung und sollten theoretisch zu einer Reduzierung des Arbeitsschutzniveaus führen.

Nach einer kurzen Diskussion der Widersprüche zwischen den Vorgaben der Verordnung und vorliegenden gesicherten arbeitswissenschaftlichen Erkenntnissen werden anhand rechnergestützter Risikobewertungen des Unfallrisikos und der Risiken gesundheitlicher und sozialer Beeinträchtigungen die mit dieser Verordnung verbundenen Absenkungen des Arbeitsschutzniveaus und die damit wiederum verbundenen Risiken für die Beschäftigten, die Patienten- und die Allgemeinversorgung demonstriert.

Die Ergebnisse zeigen, dass alle untersuchten, nach der Verordnung zulässigen Arbeitszeitmodelle mit erheblichen Risikosteigerungen für die Sicherheit und Gesundheit der Beschäftigten verbunden sind, ebenso wie mit Risiken für die Patientensicherheit und die Allgemeinversorgung. In der Diskussion wird aufgezeigt, dass die von dieser und weiteren Arbeitszeitregelungen erlaubten Massierungen der Arbeitszeit der Beschäftigten keinen erfolgversprechenden Lösungsansatz für fehlende Personalkapazitäten darstellen kann und der Arbeitszeitschutz in der BRD zurzeit keinen hohen Stellenwert hat.

Praktische Relevanz: Eine Umorientierung des Arbeitsschutzes in der BRD unter der im ArbZG gebotenen Berücksichtigung gesicherter arbeitswissenschaftlicher Erkenntnisse erscheint dringend erforderlich.

Schlüsselwörter Arbeitsschutz $\cdot$ Arbeitszeit $\cdot$ Ruhezeit $\cdot$ Unfallrisiko $\cdot$ Beeinträchtigungen

\section{Health and safety regulations concerning working time according to the FRG—using the example of the COVID-19 working time ordinance (COVID-19-ArbZV)}

\begin{abstract}
Following a short discussion concerning the contradictions of the COVID-19 working time ordinance (COVID-19-ArbZV) with the existing ergonomics evidence on the design of working time and its effects on safety, health and wellbeing possible effects of this regulation with regard to the risks of accidents, health and social impairments have been estimated using computer based risk assessments for selected working time systems constructed in accordance with the ordinance.
\end{abstract}

Dieser Artikel ist auf dem Hintergrund von drei Vorträgen zum Thema Arbeits(zeit)schutz in der BRD entstanden: einem Vortrag zur Offshore ArbZV auf dem 21. PASIG Workshop (2020), einem Vortrag zur COVID-19-ArbZV auf dem 8. Symposium der Arbeitszeitgesellschaft (2020) und auf dem PASIG Workshop (2021). Kurzfassungen der beiden PASIG-Vorträge sind im Kongressbericht des 21. Workshops (2020) und dem dazugehörigen Ergänzungsband abgedruckt.
Prof. Dr. Friedhelm Nachreiner

friedhelm.nachreiner@gawo-ev.de

1 Gesellschaft für Arbeits-, Wirtschafts- und Organisationspsychologische Forschung e. V., Achterdiek 50, 26131 Oldenburg, Deutschland 
The results show significantly increased risk estimates for all analyzed systems and for all domains, demonstrating a sincere loss in the effectiveness and efficiency of health and safety prevention. Applying these results to the medical sector leads to the conclusion of an elsewhere empirically demonstrated reduction in patient care and patient safety.

It is argued that increasing working and reducing rest times of the available work force is not an effective solution for problems of lacking human resources due to its adverse side effects on employees and third parties. Health and safety protection via working time regulations based on ergonomics evidence obviously is not a prominent approach in the FRG. Practical Relevance: A reorientation towards health and safety approaches taking ergonomics evidence into due account is required in the FRG.

Keywords Health and safety protection $\cdot$ Working time $\cdot$ Rest periods $\cdot$ Accident risk $\cdot$ Impairments

\section{Einleitung und Hintergrund}

Das Bundesministerium für Arbeit und Soziales (BMAS) hat im Einvernehmen mit dem Bundesministerium für Gesundheit (BMG) am 07.04.2020 die zeitlich begrenzt (10.04.2020 bis 30.06.2020; mit Nachwirkungen bis 31.07.2020) - und damit zum Zeitpunkt der Abfassung dieses Beitrags bereits nicht mehr - gültige COVID-19Arbeitszeitverordnung (COVID-19-ArbZV 2020) auf dem Verordnungswege zur Bewältigung der Corona-Krise erlassen. Dazu hatten sie sich am 27.03.2020 durch Einfügung des $\S 14(4)$ des Arbeitszeitgesetzes (ArbZG) (Arbeitszeitgesetz 2020) durch den Bundestag ermächtigen lassen. In der Zwischenzeit (Fassung vom 22.12.2020) ist dieser Absatz wieder entfallen. Als Begründung für diese Verordnung werden in deren Allgemeinem Teil Befürchtungen unzureichender Personalkapazitäten in der Patientenversorgung sowie in der Versorgung der Allgemeinbevölkerung angegeben. Zur Sicherstellung der Versorgung mit Hilfe erweiterter Personalkapazitäten in diesen Bereichen haben BMAS und BMG mit der COVID-19-ArbZV versucht, durch mit der Verordnung legalisierte Möglichkeiten der Ausdehnung der Arbeits- und der Verkürzung der Ruhezeiten des vorhandenen Personals in den durch die Pandemie betroffenen Bereichen - und zwar nicht nur im Bereich der Patientenversorgung - personelle Kapazitäten bereit zu stellen, auch wenn dies unter arbeitswissenschaftlicher Perspektive und auf der Basis der vorliegenden arbeitswissenschaftlichen Erkenntnisse nicht als erfolgversprechender Lösungsansatz erscheinen kann.

Die Verordnung erlaubte und legitimierte für ihren Geltungszeitraum u.a. folgende Abweichungen vom ArbZG:

- die Ausdehnung der täglichen Arbeitszeit von 8 auf $12 \mathrm{~h}$

- die Ausdehnung der wöchentlichen Arbeitszeit von 48 auf $60 \mathrm{~h}$, wobei diese $60 \mathrm{~h}$ eine Sollvorgabe (und keinen Grenzwert) darstellen, die bei Bedarf zeitlich unbegrenzt überschritten werden kann

- die Ausweitung der Ausgleichszeiten für diese Mehrarbeit über die im ArbZG vorgesehenen Zeiten hinaus
- die Reduzierung der täglichen Ruhezeit von 11 auf $9 \mathrm{~h}$, bei einem Ausgleichszeitraum von 4 Wochen

- Sonntagsarbeit, mit der Möglichkeit der Verschiebung des arbeitsfreien Sonntags,

- womit die wöchentliche Ruhezeit (ggf. sogar über einen längeren Zeitraum) entfallen kann, mit einem Ausgleichszeitraum von 8 Wochen, bzw. bis zum 31.07.2020, also einen Monat nach Auslaufen der Verordnung

Mit den damit gegebenen Möglichkeiten zur Ausdehnung und Massierung der Arbeitszeiten ließen sich völlig legal Arbeitszeiten realisieren, die den vorliegenden gesicherten arbeitswissenschaftlichen Erkenntnissen zum $\mathrm{Zu}$ sammenhang zwischen der Dauer, Lage und Dynamik von Arbeits- und Ruhezeiten und den damit verbundenen Folgen in mehrfacher Hinsicht diametral widersprechen.

Im Fokus der Erkenntnisse bei den Auswirkungen unterschiedlicher Arbeitszeitregelungen stehen dabei Aspekte der Steuerung der Effektivität und Effizienz des Arbeitshandelns und seiner Folgen, einschließlich der Arbeitssicherheit, der Gesundheit und der sozialen Teilhabe der Beschäftigten. In dem hier zur Diskussion stehenden Fall geht es darüber hinaus aber auch um direkte und indirekte Folgen dieses Arbeitshandelns und dessen Folgen für Dritte, wie beispielsweise in Form der Patientensicherheit und der Transport- und Verkehrssicherheit. Die mit der Verordnung gegebenen Möglichkeiten der Arbeitszeitgestaltung berühren damit in zentraler Form Grundgedanken effektiver und effizienter Arbeitsleistung und - insbesondere in ihren aversiven Folgen - des Arbeitsschutzes und der Wirtschaftlichkeit des Arbeitskräfteeinsatzes. Bei den vorliegenden und in diesem Kontext relevanten Erkenntnissen handelt es sich um Erkenntnisse aus experimentell kontrollierten Laborstudien, statistisch kontrollierten Feldstudien, sowie statistisch kontrollierte Sekundäranalysen von Archivdaten, ergänzt und unterstützt durch Erkenntnisse aus systematischen praktischen Beobachtungen - alle mit z.T. langer Tradition. Darüber hinaus erscheinen auch zeitlich lange vorausgehende, gleichzeitig aber vorausschauende, gesetzliche Regelungen von Interesse. 
So ist beispielsweise seit Ende des 19. Jahrhunderts (Rivers und Kraepelin 1896) bekannt, dass Arbeitsmenge und -leistung keine linearen Funktionen der Dauer der Arbeitszeit darstellen, sondern dass hier auf Grund der Rückkopplungsprozesse bei Ermüdung und Erholung (und zwischen beiden) exponentielle Zusammenhänge bestehen, ein Ergebnis, das für alle Formen körperlicher und psychischer Belastung Eingang in die einschlägige Literatur gefunden hat (z. B. im deutschsprachigen Bereich Rohmert und Rutenfranz 1983; Rutenfranz et al. 1993; Schmidtke 1965, 1993). Hinzu kommt, dass für alle Arten von Belastungen ebenfalls immer wieder gezeigt werden konnte, dass die Intensität (oder die Schwere) der Belastung in einer multiplikativen Verbindung mit ihrer Extensität (oder der zeitlichen Struktur ihrer Einwirkung) steht, so dass sich auch aus dieser Perspektive weitere überproportionale Effektsteigerungen bei den dadurch ausgelösten Beanspruchungswirkungen ergeben.

Schneider (1911) hat in einer Publikation zu den „Gefahren der Arbeit in der chemischen Industrie" bereits $\mathrm{zu}$ Beginn des vorigen Jahrhunderts darauf hingewiesen, dass nach von ihm ausgewerteten Daten über die Dauer der Arbeitszeit und die Unfallhäufigkeit in der chemischen Industrie ebenfalls ein nichtlinearer Zusammenhang zu bestehen scheint, wonach das Risiko von Unfällen in den späteren Stunden einer Schicht deutlich höher ist als in den früheren Stunden, und dies besonders bei langen (z.B. 12h) Schichten (vgl. auch ähnliche Befunde bei Teißl 1928). Diese Beobachtungen und Analysen sind in der Zwischenzeit durch statistisch kontrollierte Untersuchungen empirisch mehrfach abgesichert, wie man einschlägigen Arbeiten zu diesem Thema entnehmen kann - z. B. einer Arbeit zur Zeitabhängigkeit des Risikos meldepflichtiger Unfälle in der BRD anhand von Archivdaten der DGUV mit statistischer Kontrolle von Haupt- und Interaktionseffekten von Dauer und Lage (Haenecke et al. 1998), einem kürzlich erschienenen Review Artikel mit der Modellierung von Einzeleffekten (Fischer et al. 2017), oder einer zusammenfasenden Darstellung bei Nachreiner (2017a), mit jeweils weiteren Literaturhinweisen. Auf eine ausführliche Darstellung der umfangreichen und in sich konsistenten Literatur kann daher hier verzichtet werden.

Auf eine lange Tradition blicken auch gesetzliche Regelungen zur Gestaltung der Arbeitszeit zurück, beispielsweise in einem der ersten deutschen Arbeitsschutzgesetze, dem preußischen ,,Regulativ über die Beschäftigung jugendlicher Arbeiter in Fabriken“ von 09.03.1839 (1839). Hintergrund dieses Regulativs waren weniger humanitäre Gesichtspunkte als vielmehr die Erfahrung, dass es dem preußischen Königreich zunehmend an wehrtüchtigen jungen Männern fehlte, weil die Kinder und Jugendlichen zuvor unter Arbeits(zeit)bedingungen arbeiteten, die ihre Gesundheit und Leistungs- bzw. Wehrfähigkeit wohl erheb- lich beeinträchtigt haben. Das Regulativ regelt daher bereits sehr früh die klassischen Themen der Arbeitszeitgestaltung: Dauer (nicht länger als 10h), Lage (Beginn und Ende, keine Sonntagsarbeit) und Dauer und Lage der Pausen.

Mit den ab dem Ende des 19. Jahrhunderts einsetzenden wissenschaftlichen Untersuchungen wurden und werden in der Arbeitszeitforschung traditionell immer zwei Wirkungsketten thematisiert, die die Auswirkungen der zeitlichen Struktur der Einwirkung der Belastung (nach Dauer, Lage, Verteilung, Dynamik, Variabilität, Prognostizierbarkeit und später auch zusätzlich dem Grad der Autonomie in der Festlegung der konkreten Arbeitszeiten) vermitteln, und zwar einerseits die Wirkungskette von Ermüdung und Erholung und zweitens die Kette über die Effekte der Belegung und damit der Reduktion (normativ unterschiedlich) sozial nutzbarer Zeit durch die Lage der Arbeitszeit.

Dabei betrifft die erste Wirkungskette die mit der zeitlichen Struktur der Arbeits- und Ruhezeiten - und insbesondere deren Dynamik oder Abfolge - verbundenen Prozesse der Ermüdung und Erholung und die forderungsgerechte Erfüllbarkeit der Aufgaben- und Leistungsanforderungen, wie etwa die Quantität und Qualität der Leistung und hier insbesondere auch die Vermeidung von Handlungsfehlern und daraus potenziell oder tatsächlich resultierenden Unfällen. Entscheidend für diese Fragestellung sind die Auslenkung der an der Leistungserbringung beteiligten Funktionen des menschlichen Systems durch die während der Arbeitszeit zu bewältigende Belastung in ihrem Zeitverlauf, sowie auf der anderen Seite die Möglichkeit der Rückführung dieser Auslenkung auf das Ausgangs- oder Ruheniveau (Erholung). Kann die Auslenkung des Systems nicht innerhalb erträglicher Grenzen gehalten werden, kommt es zur Ermüdung und damit zu einer Schwächung der betroffenen Funktionen, womit eine weiterbestehende oder neu einsetzende Belastung auf verminderte Kapazitäten trifft, was zu einer verstärkten Auslenkung des Systems mit einer erhöhten Inanspruchnahme und Schwächung der verbliebenen Ressourcen (bei gleichbleibender Belastung) führt. Im Prinzip handelt es sich damit (insbesondere ab dem Überschreiten der Systemregulationsgrenzen) um einen klassischen biologischen Rückkopplungsprozess mit zwangsläufig exponentiellem Verlauf, bei der Ermüdung wie bei der Erholung; und dabei geht es um die tatsächliche, nicht um die erlebte und subjektiv bewertete Ermüdung und/oder Erholung. Als Prinzip gilt daher in der Ergonomie, dass es wichtig ist, durch Kontrolle der Intensität und/oder der Extensität der Belastung diese Systemgrenzen nicht zu überschreiten, um damit Ermüdung zu vermeiden und nicht kompensieren $\mathrm{zu}$ müssen. Graf (1922) hat sehr früh empirisch belegt, dass es ,lohnendste“ Pausen gibt, bei denen der durch die Pause eintretende Produktionsausfall während der Pausenzeit durch Mehrleistung während der verbleibenden, geringeren Arbeitszeit mehr als kompensiert wird -, auch dies wie- 
der ein Beispiel für die Nichtlinearität des Zusammenhangs zwischen der Dauer der Arbeitszeit und der Leistung.

Daher entspricht es einer alten Tradition im Arbeitsschutz, dort wo die Intensität der Belastung nicht hinreichend kontrolliert und in erträglichen Grenzen gehalten werden kann, dieses Ziel durch Kontrolle der zeitlichen Struktur ihrer Einwirkung zu erreichen. Die Ausdehnung der Extensität der Belastungseinwirkung und die (ggf. auch noch gleichzeitige) Reduzierung der Erholungszeiten kann daher weder unter Produktivitäts- noch unter Arbeitsschutzgesichtspunkten ein erfolgversprechender Ansatz sein, wie dies auch durch die vorliegende, auch neuere Literatur und auch in dem hier besonders interessierenden Kontext der Pflegearbeit, belegt wird, z. B. Blasche et al. 2017.

Die zweite Wirkungskette betrifft die Tatsache der Belegung sozial normativ für andere, insbesondere soziale, Aktivitäten vorgesehene Zeitabschnitte des Tages oder der Woche (vgl. dazu bereits Wedderburn 1981; Baer et al. 1981 sowie Ernst et al. 1983) durch Arbeitszeit und die sich daraus ergebende Interferenz von Arbeitszeit mit solchen Aktivitäten, z.B. der sozialen Teilhabe. Dabei ist empirisch belegt, dass diese Interferenz ebenfalls zu Schädigungen und Beeinträchtigungen führt, und zwar nicht nur bei den Beschäftigten selbst sondern auch bei ihren sozialen Partnern (vgl. zusammenfassend Arlinghaus und Nachreiner 2016 oder Nachreiner 2017b). Da jede Ausdehnung der Arbeitszeit die für derartige Aktivitäten verfügbare und verwertbare Zeit reduziert, müssen die Ausdehnung - und insbesondere auch die damit verbundene zeitliche Verlagerung - der Arbeitszeit zu Beeinträchtigungen führen und damit ebenfalls eine Schwächung des Arbeitsschutzes auf individueller wie auf gesellschaftlicher Ebene darstellen.

Für beide Wirkungsketten gilt, dass sie zeit(punkt)bezogen wirken. So können weder Erholung noch soziale Teilnahme auf $\mathrm{x}$-beliebige Zeitpunkte oder Zeiträume in der Zukunft verschoben werden. Körperliche wie psychische Ermüdung können nur unverzüglich, nicht aber im Abstand mehrerer Wochen kompensiert werden und soziale Teilhabe kann nur dann erfolgen, wenn die sozialen Partner ebenfalls (und zwar gleichzeitig) über dafür verfügbare Zeit verfügen; eine Tatsache, in der sich die Problematik von (insbesondere versetzten) Ausgleichszeiträumen - wie auch von Arbeitszeitkonten - deutlich wiederspiegelt. Es gilt schlicht und einfach: sich jetzt zu verausgaben und ein Jahr später zu erholen ist nicht möglich, ebenso wenig wie soziale Interaktion zu Zeiten, in denen Interaktionspartner oder soziale Angebote nicht zur Verfügung stehen.

\section{Fragestellung}

Auf der Basis dieser hier kurz skizzierten gesicherten arbeitswissenschaftlichen Erkenntnisse ergibt sich daher die
Fragestellung, ob die mit der COVID-19-ArbZV eröffneten Gestaltungsspielräume zu Arbeitszeitsystemen führen können, die mit substantiellen Erhöhungen der Risiken in Bezug auf Sicherheit, Gesundheit und soziale Teilhabe der Beschäftigten verbunden sind, in welchen Größenordnungen sich die zu erwartenden Risikoerhöhungen bewegen und ob damit substantielle Beeinträchtigungen des Arbeitsschutzes verbunden sind.

\section{Methoden}

Da bisher keine verlässlichen und validen Daten zur Umsetzung der Verordnung, der darunter realisierten Arbeitszeitsysteme oder -regelungen und ihrer Auswirkungen auf die genannten Kriterien vorliegen, haben wir unter Beachtung der durch die Verordnung gesetzten Vorgaben verschiedene zu diesem Zweck konstruierte Arbeitszeitsysteme mit Hilfe rechnergestützter Bewertungsverfahren in Bezug auf ihr Risikopotential für die oben genannten Bereiche bewertet.

Dabei handelt es sich um (zum Zeitpunkt des Erlasses der Verordnung) frei und kostenlos im Internet verfügbare Beurteilungsverfahren, und zwar um

\section{- XIMES-Risikorechner (XIMES/AUVA)}

für Forschungszwecke und Privatpersonen online kostenlos verfügbar unter https://www.ximes.com/ximesrisikorechner, sowie unter https://www.eval.at/evaluier ung-arbeitszeit

- ArbeitsZeiten Online Bewerten (AZOB, INQA)

bis vor kurzem online kostenlos verfügbar unter https:// azob.gawo-ev.de/ (bzw. als Modul in BASS 5, GAWO e. V., beide Services inzwischen eingestellt)

In beiden Fällen handelt es sich um arbeitswissenschaftlich basierte und abgesicherte Beurteilungsverfahren, die auf den Internetseiten der vom BMAS gestützten „Initiative Neue Qualität der Arbeit“ (AZOB) bzw. der Evaluationsseite der AUVA (XIMES-Risikorechner) verfügbar waren bzw. sind. Zur Beschreibung, Entwicklung und Validierung beider Verfahren verweisen wir auf die bei den Entwicklern (GAWO bzw. XIMES) jeweils angegebene Literatur.

AZOB beurteilt auf der Basis des Verlaufs von Arbeits- und arbeitsfreien Zeiten die unter einem gegebenen/ geplanten (Ist-) Arbeitszeitsystem zu erwartenden Wahrscheinlichkeiten der Beeinträchtigungen in den Bereichen Gesundheit (Schlafstörungen, Magen-Darm-Probleme) sowie soziale Teilhabe. Der XIMES-Risikorechner schätzt das unter dem gegebenen/geplanten System zu erwartende Unfallrisiko als relatives Risiko im Vergleich zu einem „normalen“ Tagarbeitssystem (tägl. Arbeitszeit von $8 \mathrm{~h}$ mit $30 \mathrm{~min}$ Pause an 5 aufeinanderfolgenden Tagen, Mo-Fr, zwischen 07 und $17 \mathrm{Uhr}$,= „Normalarbeitszeit“). Beide Systeme beschränken sich damit für ihre Risiko- 


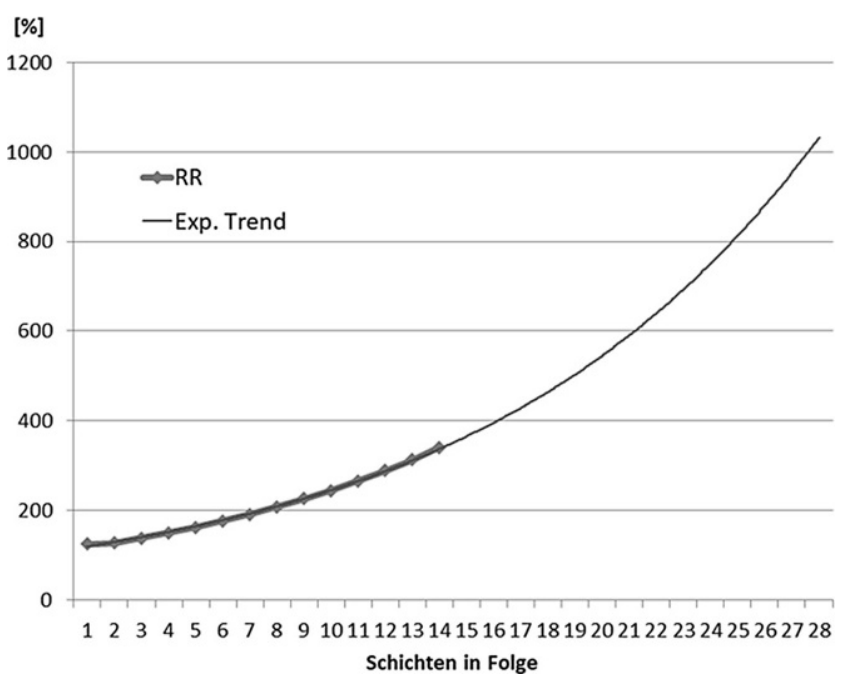

Abb. 1 Prognostizierter Anstieg des relativen Unfallrisikos (RR) über 28 12h-Tag-Schichten in Folge, unter Einhaltung einer $11 \mathrm{~h}$ Ruhezeit

Fig. 1 Predicted increase of the accident risk (RR) across $2812 \mathrm{~h}$ dayshifts in sequence, with adherence to an $11 \mathrm{~h}$ daily rest period

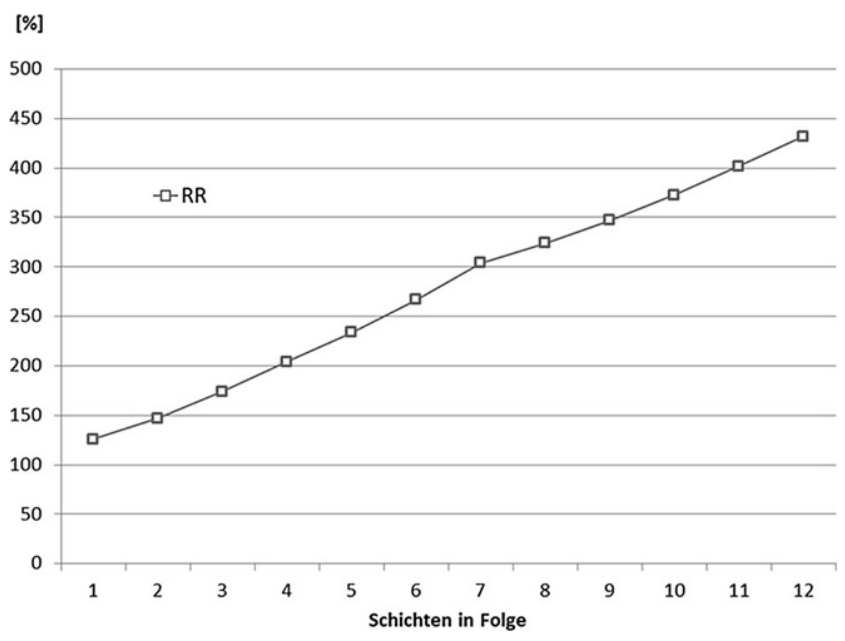

Abb. 3 Prognostizierter Anstieg des relativen Unfallrisikos (RR) über eine Folge von zwölf $12 \mathrm{~h}$ Schichten und Verkürzung der Ruhezeit auf $9 \mathrm{~h}$ in Folge innerhalb von 11 Tagen

Fig. 3 Predicted increase of the accident risk (RR) across twelve $12 \mathrm{~h}$ shifts and a reduction of the daily rest period to $9 \mathrm{~h}$ in a sequence over 11 days

beurteilung ausschließlich auf die zeitliche Struktur der Belastungseinwirkung, ohne Berücksichtigung der Intensität der Belastung oder von bestehenden Wechselwirkungen zwischen der Extensität und der Intensität der Belastung und ihrer Dynamik. Der XIMES Risikorechner ermittelt dabei ein relatives Risiko, bezogen auf die Vergleichsbasis des Mittelwertes über die ersten $8 \mathrm{~h}$ einer Schicht in der ersten Schicht der Schichtsequenz bei normaler Lage der Arbeitszeit, die gleich 100 gesetzt wird (Referenzsystem).

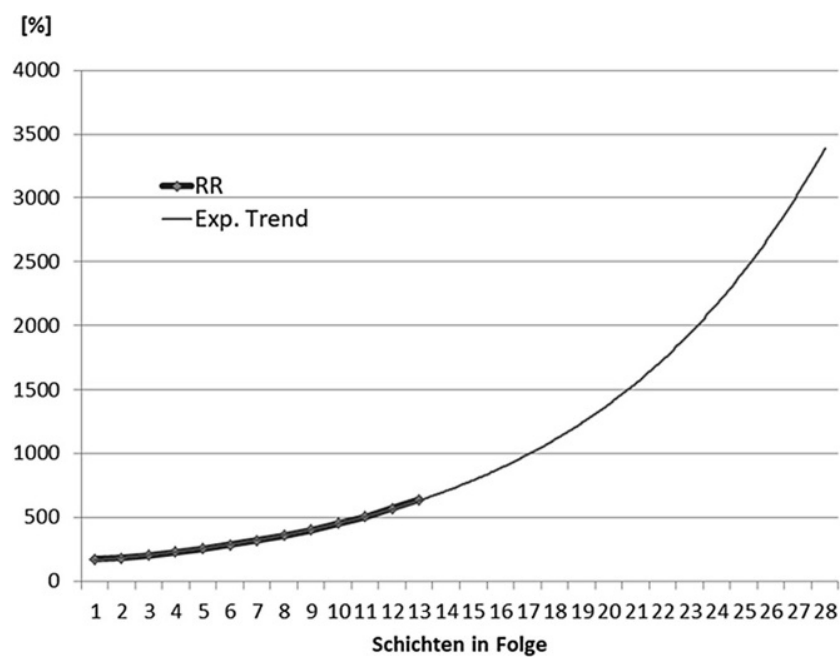

Abb. 2 Prognostizierter Anstieg des relativen Unfallrisikos (RR) über $2812 \mathrm{~h}$-Nacht-Schichten in Folge, unter Einhaltung einer $11 \mathrm{~h}$ Ruhezeit

Fig. 2 Predicted increase of the accident risk (RR) across $2812 \mathrm{~h}$ nightshifts in sequence, with adherence to an $11 \mathrm{~h}$ daily rest period

Insgesamt ist darauf hinzuweisen, dass die Güte derartiger rechnergestützter Modellierungen immer von der Qualität der Eingangsdaten (Umfang, Verteilung, modellierbare Effekte, etc.) abhängt und dass die resultierenden mittleren, von Einzeleffekten absehenden Schätzungen Abweichungen von realen Datenverläufen und ihrer konkreten Bedingungsabhängigkeit aufweisen müssen, insbesondere auch von der Art und Intensität der Belastung. Die Ergebnisse sind also nicht als Punktschätzungen für konkrete $\mathrm{Ar}$ beitsbedingungen interpretierbar - das würde die Modelle überfordern - sondern eher als Trendschätzungen der Risikoentwicklung in Abhängigkeit von der Struktur des untersuchten Arbeitszeitsystems im Vergleich zu einem Normalarbeitszeitsystem. Der Vorteil dieser Vorgehensweise besteht darin, dass damit Vergleiche zwischen verschiedenen Arbeitszeitsystemen unter Bezug auf ein Referenzarbeitssystem (Normalarbeitszeit, als beeinträchtigungsfrei unterstellt) mit diesem und untereinander - unter sonst gleichen Bedingungen - verglichen werden können.

Beurteilt wurden nach ihrer Zykluslänge differenzierte Arbeitszeitsysteme aus folgenden beiden Systemgruppen

- Arbeitszeitsysteme mit einer täglichen Arbeitszeit von $12 \mathrm{~h}, 45 \mathrm{~min}$ Pause und $11 \mathrm{~h}$ täglicher Ruhezeit, an bis zu 28 Tagen in Folge, Verschiebung der freien Sonntage auf den möglichen Ausgleichszeitraum, als Tag- bzw. als Nachtarbeit

- Arbeitszeitsysteme mit einer täglichen Arbeitszeit (oder präziser Schichtarbeitszeit) von $12 \mathrm{~h}, 45 \mathrm{~min}$ Pause, und lediglich $9 \mathrm{~h}$ täglicher Ruhezeit in Folge, über verschiedene Zeiträume, mit Ausgleich der täglichen und der wöchentlichen Ruhezeiten (durch Verschiebung der ar- 


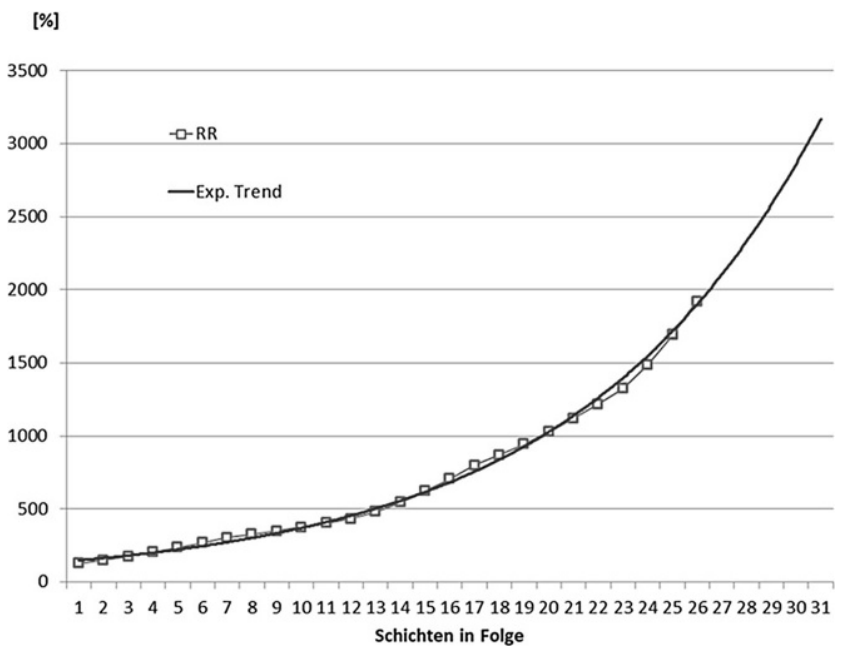

Abb. 4 Prognostizierter Anstieg des relativen Unfallrisikos (RR) über eine Folge von 31 12h-Schichten und Verkürzung der Ruhezeit auf $9 \mathrm{~h}$ in Folge innerhalb von 28 Tagen

Fig. 4 Predicted increase of the accident risk (RR) across $3112 \mathrm{~h}$-shifts and a reduction of the daily rest period to $9 \mathrm{~h}$ in a sequence over 28 days

beitsfreien Sonntage) in den jeweils vorgegebenen Ausgleichszeiträumen

Diese Systeme wurden ausgewählt, um die Grenzen des durch die Verordnung legalisierten Spielraumes auszunutzen und zu prüfen, zu welchen Risikoveränderungen die Verordnung führen kann, wenn sie denn so umgesetzt würde. Ob diese Systeme realistisch oder häufig zu erwarten waren hat dabei keine Rolle gespielt, weil es lediglich darauf ankam abzuschätzen, welche Risikoerhöhungen und damit Absenkungen des Arbeitsschutzniveaus durch die Verordnung ermöglicht wurden.

Unabhängige, experimentell manipulierte Faktoren bei diesen Analysen sind damit die Verkürzung der Ruhezeiten (Gruppe 2 vs. Gruppe 1), die Lage der Arbeitszeit (Tag- vs. Nachtarbeit/kontinuierliche kurze Rückwärtsrotation (diese genestet unter der Verkürzung der Ruhezeiten)) und die Zykluslänge der Systeme (s. Abb. 1, 2, 3, 4, 5 und 6). Dabei wurde bewusst in Kauf genommen, dass die Systeme mit verkürzten Ruhezeiten zu sog. „Doppelschlägen“ (also 2 Schichtbeginnen innerhalb eines Arbeitstages) führen, mit der offenen Problematik der Bestimmung der täglichen Arbeitszeit (nach ArbZG „Beginn bis zum Ende der Arbeitszeit").

Vergleiche mit anderen nach dem ArbZG (mit und ohne Ausnahmegenehmigungen) zulässigen Arbeitszeitsystemen sind zwar möglich - Risikoschätzungen für unterschiedlichste (reale und geplante) Schichtsysteme liegen vor, sind publiziert und lassen sich beliebig erstellen - erscheinen in dem hier interessierenden Zusammenhang nicht relevant, weil es hier nicht darum geht, die (Grenzen der) Schutzgüte des ArbZG mit seinen zahlreichen Ausnahmeregelungen zu überprüfen, sondern um Fragen der Verletzung der Ausführbarkeit und der Beeinträchtigungsfreiheit (als arbeitswissenschaftliche Arbeitsschutzziele) der durch die Verordnung legalisierten Systeme.

Der guten Ordnung halber soll darauf hingewiesen werden, dass gängige Schichtsysteme in Produktion und Dienstleistung, die entgegen § 6(1) ArbZG nicht die gesicherten arbeitswissenschaftlichen Erkenntnisse berücksichtigen (z.B. lang rückwärts rotierte Konti-Systeme), eben wegen dieser Nichtberücksichtigung nicht ArbZG-konform sind und daher für Vergleichszwecke wenig geeignet erscheinen.

\section{Ergebnisse}

Alle durchgeführten Bewertungen aller untersuchten Arbeitszeitsysteme zeigen, wie zu erwarten, erhebliche Erhöhungen der Risiken für Sicherheit, Gesundheit und soziale Teilhabe.

\subsection{Prognose des Unfallrisikos}

Abb. 1 zeigt den prognostizierten Anstieg des Unfallrisikos für ein System mit $12 \mathrm{~h}$ täglicher Arbeitszeit, einer Pause von $45 \mathrm{~min}$ in der Mitte der Schicht und Einhaltung der gesetzlich vorgegebenen täglichen Ruhezeit von $11 \mathrm{~h}$. Die Samstage und Sonntage sind ebenfalls Arbeitstage, eine wöchentliche Ruhezeit ist nicht gegeben. Die dickere Linie mit den Rauten markiert dabei die numerischen Ergebnisse des Risikorechners bis zur 14. Schicht in Folge, während die dünnere Linie den daraus extrapolierten exponentiellen Trend kennzeichnet. Dieses Vorgehen war erforderlich, weil der XIMES-Rechner das Risiko nach oben begrenzt und damit jenseits dieser Grenzen keine präzisen und validen Schätzungen mehr erlaubt. Grund für diese Begrenzung ist einerseits die Beschränkung auf Arbeitszeitsysteme, die über das vier- bis fünffache Risikos eines Standardsystems, also eines $5 \times 8 \mathrm{~h}$ Tagarbeitssystems, nicht hinausgehen, weil dies aus arbeitswissenschaftlicher Sicht bereits über das verantwortbare Risiko hinausgeht und zwingend zu Änderungen des Arbeitszeitsystems führen müsste. Darüber hinaus ist die Datengrundlage für eine Risikomodellierung solch extremer, aber nach der Verordnung zulässiger, Arbeitszeitmodelle sehr begrenzt.

Wie aus Abb. 1 erkennbar, wird bereits für die erste $12 \mathrm{~h}$ Schicht eine Erhöhung des schichtbezogenen Unfallrisikos um $27 \%$ erreicht. Dabei liegt das Risiko für die 12. Stunde in dieser Schicht etwa beim Doppelten des durchschnittlichen Risikos der ersten $8 \mathrm{~h}$ (hier nicht dargestellt, vgl. dazu die auf tatsächlichen Daten basierenden Ergebnisse in der Literatur, z. B. bei Haenecke et al. 1998; mit Differenzierung nach verschiedenen Schichten, oder allgemein Nach- 


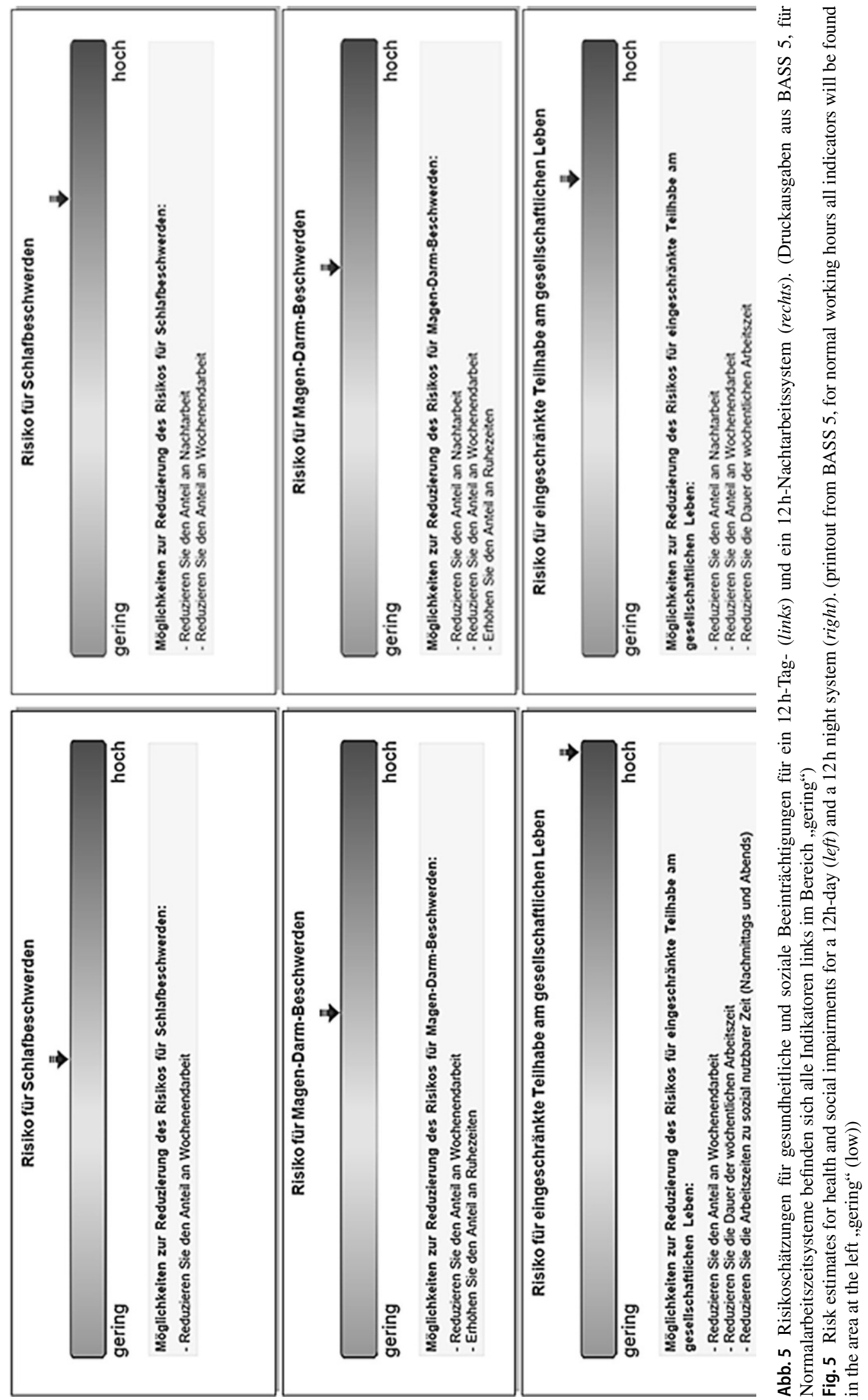


Abb. 6 Risikoschätzung für gesundheitliche und soziale Beeinträchtigungen für ein $12 \mathrm{~h}$ Arbeitszeitsystem mit 9h Ruhezeit, 23 Schichten an 21 Tagen in Folge

Fig. 6 Risk estimates for health and social impairments for a $12 \mathrm{~h}$-shiftsystem with $9 \mathrm{~h}$ daily rest, 23 shifts in sequence within 21 days

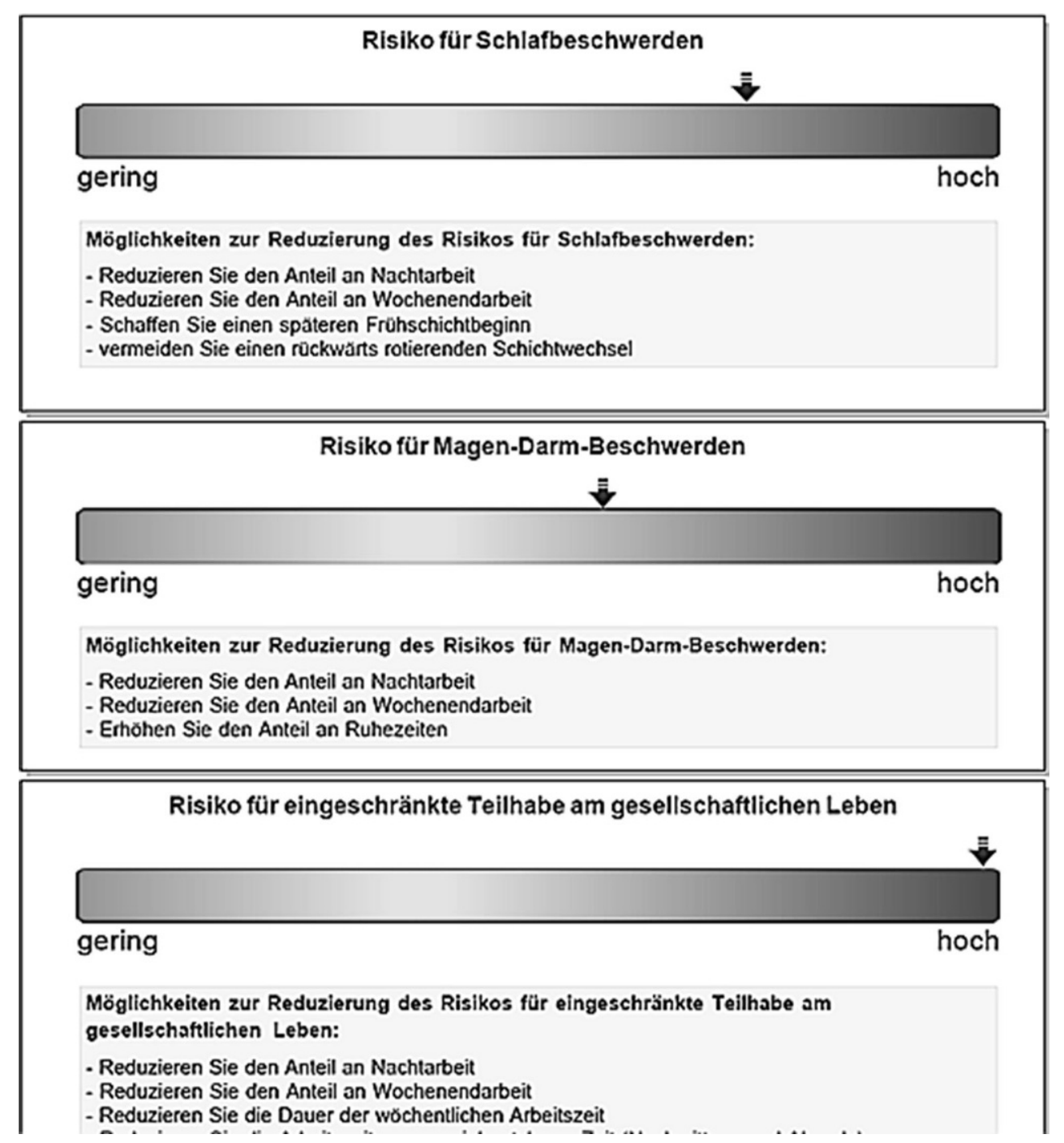

reiner 2017a). Nach sieben solcher $12 \mathrm{~h}$ Schichten in Folge wird bereits eine Verdopplung des schichtdurchschnittlichen Unfallrisikos im Vergleich mit einem Standardarbeitszeitsystem erreicht. Nach 16 solcher Schichten ergibt sich eine Risikoschätzung von $400 \%$ des Vergleichsrisikos, und damit eine Vervierfachung des Risikos im Tagesmittel (mit den jeweils entsprechenden Erhöhungen in den letzten Arbeitsstunden der Schicht), nach 28 Tagen wird mit $1000 \%$ das zehnfache Risiko im Schichtdurchschnitt erreicht.

Abb. 2 zeigt die Risikoschätzungen für dasselbe Arbeitszeitsystem bei Nachtschicht. Deutlich ist auch hier wieder der gleiche exponentielle Trend zu beobachten, hier allerdings wegen der Lageverschiebung der Arbeitszeit in die Nacht mit einem höheren Ausgangswert bereits für die erste Nacht und einem stärkeren Anstieg über die folgenden Nächte. So ergibt sich bereits in der fünften $12 \mathrm{~h} \mathrm{Nacht-}$ schicht in Folge eine Risikosteigerung auf das Doppelte des Vergleichsstandards, das zehnfache Risiko wird in der 17. Schicht in Folge erreicht und nach 28 Nachtschichten in
Folge ergibt sich eine Risikoschätzung von nahezu 3500\%, also das 35 fache Risiko im Schichtdurchschnitt, jeweils mit den entsprechenden Erhöhungen dieser Werte in den jeweils letzten Stunden dieser Schichten.

Abb. 3 zeigt die Risikoschätzungen für ein System mit der Kombination der Ausdehnung der Arbeitszeit auf 12h, plus einer 45-minütigen Pause, und der Verkürzung der folgenden Ruhezeit auf 9h, und zwar für ein System mit 12 Schichten an 11 Tagen, was durch die kurzen Wechsel zwischen den Schichten bedingt ist. Deutlich wird hier der noch schnellere Risikoanstieg über die Schichten hinweg, wobei die Verdopplung des schichtbezogenen Risikos bereits in der vierten Schicht in Folge erreicht wird. Deutlich wird auch der steilere Anstieg des Risikos über die ersten sieben im Vergleich $\mathrm{zu}$ den folgenden fünf Schichten, weil die ersteren Nachtanteile und die letzteren Tagarbeit beinhalten (was gleichzeitig die Sensitivität und Validität des Beurteilungsinstrumentes demonstriert), wobei sich für 
die Teilabschnitte unterschiedliche Exponentialfunktionen ergeben.

Abb. 4 macht deutlich, dass mit dieser Massierung der Arbeitszeit in diesem Modell mit $12 \mathrm{~h}$ Schichten (incl. Nachtschichten) und verkürzten Ruhezeiten über einen Schichtzyklus von 28 Tagen (und dabei 31 Schichten in Folge) eine Steigerung des relativen schichtbezogenen Unfallrisikos auf rund $3000 \%$ im Vergleich zum Standardsystem zu erwarten ist. Dabei wird wiederum der unterschiedliche Anstieg über die Tag- und die Nachtarbeitsanteile deutlich erkennbar. Dehnte man dieses Arbeitszeitmodell auf sechs Wochen (d.h. 46 Schichten an 42 Tagen, Verschiebung der fehlenden Ruhezeiten in den Ausgleichszeitraum) aus, wäre eine Risikosteigerung um $14.000 \%$, also das 140 fache des Standard-Vergleichsmodells zu erwarten. Dies würde allerdings zu Problemen mit dem Ausgleich der täglichen Ruhezeiten führen, da der Ausgleichszeitraum dafür auf vier Wochen begrenzt ist; Abhilfe könnte dabei ein Verzicht auf einige der letzten Schichten oder der Verzicht auf „Doppelschläge“ (zwei Schichtbeginne am selben Tag) bieten, womit der formale Ausgleich und gleichzeitig eine (unzureichende) Risikoabsenkung erreicht werden könnte.

\subsection{Beurteilung des Risikos für gesundheitliche und soziale Beeinträchtigungen}

Betrachtet man die mit solchen Arbeitszeitregelungen verbundenen Risiken für gesundheitliche und soziale Beeinträchtigungen, so lassen sich auch hier erhebliche Risikosteigerungen gegenüber Normalarbeitszeitsystemen $(5 \times 8 \mathrm{~h}$ Tagarbeit, Mo-Fr) erkennen. Abb. 5a zeigt die Ergebnisse für ein 12h-Tagsystem (08:00 bis 20:45, mit einer 45-minütigen Pause in der Schichtmitte) über einen Zeitraum von 4 Wochen.

Wie man aus Abb. 5 erkennen kann, ergeben sich bei Tagarbeit deutlich erhöhte Risiken für Schlafbeschwerden, einem der zentralen Indikatoren gesundheitlicher Beeinträchtigungen durch die Arbeitszeitgestaltung (auch wenn der Nachtschlaf durch die Lage der Arbeitszeit kaum gestört sein dürfte), wie auch bei den Magen-Darm-Beschwerden, beide vergleichbar einem suboptimal gestalteten Schichtsystem. Auffallend ist hier vor allem aber das Risiko einer eingeschränkten Teilhabe am gesellschaftlichen Leben, die die Grenzen der Beurteilungsskala erreicht. Hier ergibt sich ein ähnliches Problem wie bei dem XIMES-Risikorechner; auch AZOB bzw. BASS 5 sind im Wesentlichen für „normale“ Beurteilungsfälle ausgelegt und nicht für die mit der Verordnung legitimierten Ausnahmeregelungen. Dennoch lassen die Ergebnisse erkennen, dass die Skala der als möglich erachteten Risikosteigerungen ausgeschöpft wird, bei diesem Tagsystem insbesondere durch die Lage der Arbeitszeit, die soziale Kontakte nahezu unmöglich macht, weil sie die dafür normativ vorgesehenen Zeiten überdeckt.
Für das Nachtarbeitssystem (20:00 bis 08:45) mit ansonsten vergleichbarer Struktur der Arbeitszeiten ergibt sich erwartungsgemäß eine weitere deutliche Steigerung des Risikos für Schlafbeschwerden. Verbunden mit dieser Lage der Arbeitszeit, die mehr nutzbare Zeiten für soziale Interaktionen belässt, zeigt sich eine Verringerung des Risikos gegenüber dem Tagarbeitssystem, dennoch bleibt die Erhöhung gegenüber einem Normalarbeitszeitsystem (alle Risikoindikatoren $(\downarrow)$ im Bereich ,gering“, hier nicht dargestellt) erheblich und beachtlich.

Abb. 6 zeigt die Risikoabschätzungen für gesundheitliche und soziale Beeinträchtigungen für ein $12 \mathrm{~h}$-Arbeitszeit/9h-Ruhezeitsystem für einen Zeitraum von 3 Wochen. Deutlich erkennbar sind wieder Risikoerhöhungen bei den zentralen Merkmalen Schlafstörungen und soziale Beeinträchtigungen; auch dieses Arbeitszeitsystem stößt in diesen Bereichen wieder an die Grenzen des Beurteilungssystems.

\section{Diskussion}

Wie die Ergebnisse unserer Modellsimulationen zeigen, ermöglicht und legalisierte die COVID-19-ArbZV des BMAS Arbeitszeitsysteme, die als höchst risikobehaftet und inhuman zu bewerten sind, und zwar im Hinblick auf sämtliche arbeitswissenschaftliche Kriterien humaner Arbeitsgestaltung: Ausführbarkeit, Schädigungslosigkeit, wie Beeinträchtigungsfreiheit. Die mangelnde (forderungsgerechte) Ausführbarkeit der Arbeit unter den legalisierten Arbeitszeitbedingungen zeigt sich in der drastischen und nicht hinnehmbaren Erhöhung des Unfallrisikos unter allen untersuchten Arbeitszeitsystemen. Die mangelnde Schädigungslosigkeit zeigt sich, neben dem erhöhten Unfallrisiko, in der substantiellen Erhöhung der Risiken gesundheitlicher Beeinträchtigungen, hier operationalisiert an den ausgewählten und in der Bewertung von Arbeitszeitsystemen bewährten Indikatoren Schlafstörungen und Magen-Darm-Probleme. Weitere Beeinträchtigungen, verbunden mit potenziellen Schädigungen, lassen sich erkennen in der Erhöhung der Risiken für soziale Beeinträchtigungen, die nicht nur die Beschäftigten selbst sondern auch deren (primäres wie sekundäres) soziales Umfeld (Familienmitglieder, Freunde und Bekannte) treffen. Die durch diese Systeme zu befürchtenden Beeinträchtigungen entfalten damit Wirkungen, die weit über die von der Verordnung direkt Betroffenen hinausgehen. Bei der damit gegebenen und aus arbeitswissenschaftlicher Sicht nicht hinnehmbaren Nichterfüllung auch nur der Defizitkriterien (Vermeidung aversiver Beanspruchungs- und damit Belastungsfolgen) erübrigt sich die Prüfung und Diskussion einer potenziellen Erfüllung positiver oder Wachstumskriterien wie Lern- oder Persönlichkeitsförderlichkeit von selbst und kann hier entfallen. Unter der 
Perspektive des Arbeitsschutzes kann die Verordnung nur als dysfunktional und kontraproduktiv beurteilt werden.

Aber auch die intendierten Effekte der Verordnung auf andere Kreise als die der Beschäftigten, die ja das eigentliche Ziel der Verordnung darstellen, erscheinen unter einer arbeitswissenschaftlichen Perspektive durch die Effekte auf die Beschäftigten bedroht, wenn nicht gar unmöglich gemacht. Eines dieser Ziele war ja u.a. die Aufrechterhaltung - oder gar die Verbesserung - der Patientenversorgung und der Patientensicherheit während der Pandemie. Berücksichtigt man, dass auch das medizinische Personal den einleitend vorgestellten Zusammenhängen zwischen Arbeits(zeit)gestaltung und deren Effekten unterliegt, so ist auch hier unter den von der Verordnung legalisierten Arbeitszeitsystemen bei der erreichbaren Ausdehnung von Arbeitszeiten, der dadurch direkt bedingten Reduzierung der Erholungszeiten, und der per Verordnung legitimierten weiteren Reduzierung der Erholungszeiten durch die Möglichkeit zur Verkürzung und Verschiebung der Ruhezeiten, und damit der Möglichkeiten einer erheblichen Massierung der Arbeitszeiten, mit einer exponentiellen Steigerung des Risikos für medizinische oder pflegerischen Fehlhandlungen zu rechnen. Wie ein Blick in die einschlägige Literatur (z.B. zusammenfassend bis 2010 Nachreiner et al. 2010; für neuere Literatur siehe u. a. Härmä et al. 2020; mit jeweils weiteren Nachweisen) zeigt, ist dies nicht nur zu erwarten sondern empirisch inzwischen gut belegt, obwohl es sich dabei um ein tabuisiertes und daher nur schwer zugängliches Forschungsthema handelt. Belegt sind dabei Zunahmen aller Arten von Handlungsfehlern, von falschen Medikationen über Fehlentscheidungen bis zu Fehlern bei operativen Eingriffen. Aber auch sporadische und - auch auf Nachfrage - nicht durch gesicherte Forschungsergebnisse belegte Erfahrungsberichte in der Presse (Rinke 2020) weisen darauf hin, dass die Lage im Klinikum in Wuhan, dem Ausbruchsort des für COVID-19 verantwortlichen Virus, angeblich erst unter Kontrolle gebracht werden konnte, nachdem die Schichtdauer des Personals auf 6 (!) Stunden herabgesetzt wurde.

Lange Arbeitszeiten sind danach auch im Krankenhausbereich, nach der vorliegenden Befundlage wie nach unkontrollierten Erfahrungsberichten, mit häufigeren Fehlentscheidungen und Fehlhandlungen verbunden und führen zu einer Verringerung der Qualität der Patientenversorgung und der Patientensicherheit. Die COVID-19-ArbZV dürfte damit wohl auch nicht geeignet (gewesen) sein, ihre ureigensten Zielvorstellungen zu erreichen; im Gegenteil dürften die durch die Verordnung provozierten und legalisierten Arbeitszeitregelungen bei ihrer Anwendung zu einer Verschlechterung der Patientenversorgung und der Patientensicherheit geführt haben. Mit den nach arbeitswissenschaftlichen Erkenntnissen zu erwartenden Effekten in diesem Bereich, wie auch in dem der Allgemeinversorgung lässt sich aus arbeitswissenschaftlicher Perspektive der Erlass der Verordnung also nicht legitimieren. So haben sich beispielsweise auch im Kontext des Verkehrswesens und der Verkehrssicherheit lange Arbeitszeiten (neben langen Lenkzeiten, die aber in der Verordnung nicht angetastet wurden) als dysfunktional erwiesen. Der Erlass von Verordnungen oder Allgemeinverfügungen mit derartigen Inhalten kann aus arbeitswissenschaftlicher Sicht auch nicht legitimiert werden, wenn - wie in der Allgemeinverfügung des Niedersächsischen Sozialministeriums (2020), die ebenfalls die maximale tägliche Arbeitszeit auf $12 \mathrm{~h}$ erhöht und Sonntagsarbeit mit Verschiebung der wöchentlichen Ruhezeiten legitimiert - darauf hingewiesen wird, dass individueller Arbeitsschutz zugunsten des Gemeinwohls zurückstehen müsse; ein Gedanke, dem man näher treten könnte, wenn denn Effektivität und Effizienz solcher Maßnahmen in Hinsicht auf die Zielstellung nachgewiesen oder auch nur zu erwarten wären - was sie nach den vorliegenden arbeitswissenschaftlichen Erkenntnissen jedoch nicht sind, auch nicht in Krisenzeiten (vgl. dazu die Berichte über Erfahrungen mit langen Arbeitszeiten in der englischen Munitionsindustrie im 1. Weltkrieg bei Vernon 1921).

Die - in der Zwischenzeit ausgelaufene - Verordnung hätte unter der im ArbZG gebotenen Berücksichtigung arbeitswissenschaftlicher Erkenntnisse (die bisher nicht aufgehoben wurde) nie erlassen werden dürfen und sollte daher - auch in ihrem Ansatz - für immer zurückgezogen werden. Sie sollte inhaltlich auch nie wieder reaktiviert werden oder, wie jetzt, in verschiedenen Bundesländern (ebenfalls unter Nichtberücksichtigung der vorliegenden arbeitswissenschaftlichen Erkenntnisse) in Allgemeinverfügungen umgesetzt werden können, auch nicht in ihren Teilvorgaben. Man sollte vielleicht bei solchen Überlegungen im Hinterkopf behalten, dass das ArbZG zwar im Wesentlichen ein Gesetz zum Schutz der Beschäftigten darstellt, bei dessen Entwicklung allerdings auch (volks-) wirtschaftliche und gesamtgesellschaftliche Überlegungen im Hintergrund gestanden haben, wie beispielsweise bereits im preußischen Regulativ von 1839.

Es sollte allerdings auch berücksichtigt werden, dass die hier berichteten Probleme mit den Abweichungen von gesicherten arbeitswissenschaftlichen Erkenntnissen in der BRD ja nicht singulär sind, z.B. nur im Rahmen einer Pandemie, sondern durchaus auch in anderen Bereichen und unter normalen Betriebsbedingungen vorzufinden sind, und zwar im Wesentlichen aus wirtschaftlichen oder verwaltungstechnischen Gründen, beispielsweise im Offshore - Bereich. Hier erlaubt die vom BMAS erlassene Offshore-Arbeitszeitverordnung (Offshore-ArbZV 2013) ebenfalls gravierende Abweichungen vom Arbeitszeitgesetz, die mit den gesicherten arbeitswissenschaftlichen Erkenntnissen nicht in Einklang stehen, u.a. (etwas vergröbert) $12 \mathrm{~h}$-Schichten über 14 Tage in Folge offshore, 
gefolgt von 14 zusammenhängenden Tagen Ruhezeit an Land. Auch hier zeigen Risikoschätzungen, wie nicht anders zu erwarten, eine exponentielle Zunahme des Risikos im Schichtverlauf und über den Schichtzyklus hinweg (vgl. Nachreiner 2020; Arlinghaus und Gärtner 2020). Bei den dort gefundenen Risikosteigerungen kommt hinzu, dass die Beurteilungsmodelle von „,normalen“ Erholungsbedingungen und Bedingungen für soziale Teilhabe ausgehen, was aber bei offshore-Arbeit offensichtlich nicht der Fall ist. Unsere Risikoschätzungen stellen damit auch hier eher zu konservative Schätzungen der zu erwartenden Beeinträchtigungen dar. Anzumerken bleibt, dass durch die Legalisierung solcher Arbeitszeitsysteme durch diese Verordnung des BMAS der Handlungsspielraum von Betriebsräten bei Versuchen zur Durchsetzung beeinträchtigungsminimierender Arbeitszeitsysteme, z. B. auch in Einigungsstellen, in erheblichem Maße eingeschränkt wird.

Berücksichtigt man ferner, dass unsere Risikoschätzungen ausschließlich auf Modellen beruhen, die lediglich die zeitliche Struktur der Belastungseinwirkung modellieren, Art und Intensität der Belastung - insbesondere auch in ihrer Interaktion mit der zeitlichen Struktur der Einwirkung - jedoch (noch) unberücksichtigt lassen und sich auf eine mittlere Belastung beziehen, wird man wiederum erkennen, dass die derzeitig vorliegenden Instrumente die gegebenen Risiken deutlich unterschätzen, die hier vorgelegten Schätzungen also in der Tat ausgesprochen konservativ sind. Höchstwahrscheinlich müssen wir daher von erheblich größeren Risiken ausgehen, insbesondere bei extremen Belastungen, wie sie etwa im Kontext der Arbeit im Gesundheitswesen während einer Pandemie narrativ berichtet werden.

Limitationen der hier vorgestellten Studie bestehen in der bereits erwähnten begrenzten Qualität der Basisdaten für die Modellierung und Validierung der Modelle. Geeignete, objektive Daten sind im Gegensatz zu Meinungsumfragen, die aber an den entscheidenden Stellen nicht differenzieren können, nur selten vorhanden und dann auch noch verfügbar. Die Modellierung wird ggf. weiter begrenzt durch fehlende Angaben zu den Bedingungskonstellationen oder die Anzahl der Fälle je relevanter Kombination wird schnell relativ klein, mit entsprechenden Vergrößerungen der Schätzfehler. Daher sind weitere Validierungsuntersuchungen der vorliegenden Modelle dringend erforderlich. Für die hier eingesetzten Verfahren liegen entsprechende Validierungsuntersuchungen, sogar als Kreuzvalidierungsuntersuchungen, mit befriedigenden Ergebnissen vor (vgl. die Angaben zu beiden Instrumenten in der Literatur, z. B. Schomann et al. 2006; Giebel et al. 2009). Dennoch würde man sich in dem vorliegenden Fall der nahezu kompletten Aussetzung von Schutzvorschriften bei den theoretisch wie praktisch zu erwartenden Effekten schon aus ethischen Gründen keine spezifische Validierung der Prognose der aversiven Effekte wünschen - die mit der Verordnung legalisierten Arbeitszeitmodelle liegen schließlich außerhalb des arbeitswissenschaftlich vorstellbaren Denkraumes.

Wenn aber eine solche aus arbeitswissenschaftlicher Sicht völlig inakzeptable Verordnung erlassen wird, wäre allerdings zumindest eine begleitende arbeitswissenschaftliche Untersuchung des Umfangs der Umsetzung der Ausnahmen in der Verordnung (nach den Merkmalen der konkreten Ausdehnung der Arbeits- und Reduzierung der Ruhezeiten nach Umfang und Häufigkeit), verbunden mit einer Analyse der tatsächlichen Auswirkungen sehr wünschenswert gewesen. Eine solche Untersuchung wäre aber allein schon wegen der zeitlichen Bedingungen bei der Umsetzung der Verordnung weder von der Planung noch von der Umsetzung einer validen Untersuchung her durchführbar gewesen. Dabei muss auch als Problem betrachtet werden, dass die Verordnung die Betriebe nicht verpflichtet hat, die Umsetzung (und schon gar nicht nach differenzierten Merkmalen) zu dokumentieren. Daher liegen keine validen Daten vor, die man für eine solche Validierung der Modelle und ihrer Prognosen oder der Abschätzung der tatsächlichen Effekte nutzen könnte. Ob sich im Rahmen der von der EU verpflichtend vorgegebenen - aber wohl bisher in der BRD nicht konsequent umgesetzten - Erfassung der konkreten Arbeitszeiten bessere Bedingungen für eine nachträgliche Validierung der Effekte und ihrer Prognosen ergeben, bleibt abzuwarten. Ähnliches gilt für die Erfassung der Effektvariablen. Wir werden uns daher bis auf weiteres mit der bisher nachgewiesenen Validität der Verfahren begnügen und entsprechende Vorsicht walten lassen müssen. (Quasi-) Experimentell kontrollierte Untersuchungen in diesem Problemkontext in der betrieblichen Praxis verbieten sich dagegen bei den zu erwartenden aversiven Konsequenzen jedoch aus ethischen Gründen schon von selbst.

Trotz der beschriebenen Limitationen sollten die vorgestellten Ergebnisse und Überlegungen Entscheidungsträger davon abhalten, bei der Lösung konkreter Probleme auf unzureichend reflektierte Lösungsansätze zurückzugreifen. Vielmehr sollten wir versuchen, alle vorhandenen relevanten Erkenntnisse zu nutzen, selbst wenn diese nicht perfekt erscheinen mögen, um prognostizierbar suboptimale oder dysfunktionale Lösungen zu vermeiden. Für den hier gegebenen Fall des Versuchs, eine Lösung über die Veränderung der Arbeitszeiten zu erreichen, wären daher zunächst inhaltlich begründete Abschätzungen des Arbeitskräftebedarfes und seiner zeitlichen Dynamik erforderlich gewesen, um darüber mit den vorhandenen Modellen und Instrumenten der Arbeitszeitgestaltung betriebsbezogene (und keine allgemeinbezogenen) Lösungen unter Berücksichtigung der jeweiligs vorhandenen oder realistich zu erwartenden Möglichkeiten zur Erweiterung der personellen Ressourcen unter Einhaltung der arbeitswissenschaftlichen Erkenntnisse 
zu entwickeln. Dabei wäre sehr schnell deutlich geworden, ob, wie und in welchem Umfang eine sozialverträgliche Lösung mit Mitteln der Arbeitszeitgestaltung möglich gewesen wäre, am ehesten wohl noch über Ansätze zur Verkürzung von Arbeitszeiten und Einhaltung der gegebenen Mindestruhezeiten. Dabei wären auch sehr schnell die Grenzen von zeitlichen Umgestaltungsmaßnahmen erkennbar geworden, z. B. wenn die verfügbaren personellen Ressourcen innerhalb verantwortbarer Grenzen erschöpft wären und jede weitere Ausdehnung der Nutzung zu deren Abbau führen müsste.

Wir sollten ferner dringend versuchen, unsere Methoden und Erkenntnisse zu verbessern, nach oben ist hier noch jede Menge Spielraum. Dies würde allerdings personelle und finanzielle Investitionen erfordern, z. B. bei der Modellierung von Erholung und (Unfall- oder Fehlhandlungs-) Risikoverläufen während der arbeitsfreien Zeit und der Effekte variabler Arbeitszeiten (Nachreiner et al. 2019). Dabei sollten wir uns auch wieder stärker auf faktenbezogene Daten beziehen (wie etwa die konkreten Ist-Arbeitszeiten auf der unabhängigen und Fehlhandlungen oder Unfälle auf der abhängigen Seite) und uns weniger auf kognitiv und emotional ggf. verzerrte subjektive Beurteilungen dieser Bedingungen verlassen (wie etwa erlebte Ermüdung und erlebte Erholung, im Gegensatz zur tatsächlichen Funktionsauslenkung und -rückführung), auch wenn diese einfacher zu erheben, dafür aber bekanntermaßen weniger valide sind - vgl. die bekannten Paradoxien der Ermüdung (Hacker und Richter 1980).

Insgesamt erscheint damit der Arbeits(zeit)schutz in der BRD verbesserungsfähig und verbesserungswürdig, zumindest aber erhaltenswert. So weisen die hier vorgelegten Ergebnisse darauf hin, dass einige der Regelungen im ArbZG aus Arbeitsschutzgründen nicht aufgegeben werden sollten, auch nicht im Rahmen einer von bestimmten Interessenvertretern favorisierten Liberalisierung der entsprechenden Vorgaben bei einer Revision des derzeitigen ArbZG. Ob das derzeitige ArbZG (mit oder ohne seine zahlreichen Ausnahmeregelungen) tatsächlich in der Lage ist, wie auch in unseren Berechnungen der Einfachheit halber unterstellt, Ausführbarkeit, Gesundheit und Beeinträchtigungsfreiheit zu garantieren, soll hier dahingestellt bleiben. Unsere eigenen wissenschaftlichen wie praktischen Erfahrungen (z.B. in Einigungsstellen) mit nach dem ArbZG zulässigen in der betrieblichen Praxis verfahrenen Arbeitszeitsystemen lassen daran erhebliche Zweifel aufkommen. Das ArbZG und intendierte oder geforderte Revisionen sollten daher gründlich auf ihre Sozialverträglichkeit geprüft werden, auch unter Einsatz rechnergestützter Bewertungsinstrumente, bevor solche Änderungen legalisiert und den Beschäftigten zugemutet werden. Dabei sind dann auch langfristige Effekte der Arbeitszeitgestaltung, wie etwa eine vorzeitige Berufs- oder Erwerbsunfähigkeit, zu berücksichtigen (vgl. etwa Bockel- mann et al. 2016; Wirtz und Nachreiner 2012), die bei der COVID-19-ArbZV wegen ihrer zeitlich begrenzten Gültigkeit nicht von primärer Bedeutung erscheinen, dies bei anderen Veränderung der normativen Vorgaben, wie beispielsweise bei der Offshore-ArbZV, aber durchaus sein können.

Der bestehende Arbeits(zeit)schutz in der BRD hat bereits jetzt offensichtlich keinen hohen Stellenwert, insbesondere bei konkurrierenden Interessen, wie die hier und an anderer Stelle (Nachreiner 2020) vorgestellten Ergebnisse zeigen. Dies zeigt auch die Behandlung der Arbeitszeit in den vom BMAS vorgelegten SARS-CoV-2-Arbeitsschutzstandard (2020) und SARS-CoV-2-Arbeitsschutzregel (2020) - von der Umsetzung der bestehenden normativen Arbeitszeitvorgaben und ihrer Kontrolle durch die zuständigen Aufsichtsbehörden, vgl. etwa den Bericht des Arbeitsministeriums von NRW zur Situation in der Fleischindustrie (MAGS NRW 2019) oder den Bericht über die tendenziell rückläufige Kontrollhäufigkeit von Betrieben durch die Aufsichtsbehörden (BMAS 2020) einmal ganz abgesehen. In den oben aufgeführten SARS-CoV-2 - Regelungen beschränkt sich der Arbeitszeitschutz im Wesentlichen auf die Regelung der Beschränkung der Kontakte bei den Schichtübergaben. Dass die Dauer der Arbeitszeit (in Wechselwirkung mit der Art der Tätigkeit) ebenfalls in einem $\mathrm{Zu}$ sammenhang mit der Übertragungswahrscheinlichkeit stehen dürfte, erscheint dagegen weniger relevant. Konsistent damit ist ein jüngst im Auftrag des BMAS erstellter Bericht (Bonin et al. 2021) zur Wirksamkeit der Corona-Maßnahmen des BMAS: die COVID-19-ArbZV kommt darin nicht vor.

\section{Literatur}

Allgemeinverfügung zur Durchführung des Arbeitszeitgesetzes - ArbZG - Ausnahmebewilligung zur Beschäftigung von Arbeitnehmerinnen und Arbeitnehmern an Sonn- und Feiertagen und für Abweichungen von bestimmten Beschränkungen des ArbZG aus Anlass der Ausbreitung des Corona-Virus (SARS-CoV-2) in Deutschland gemäß $§ 15$ Abs.2 ArbZG / AV d. MS v. 21. Okt. 2020 - 40012/1-15-02-, Nds. MB1. Nr. 49/2020

Arlinghaus A, Nachreiner F (2016) Unusual and unsocial? Effects of shift work and other unusual working times on social participation. In: Iskra-Golec I, Barnes-Farell J, Bohle P (Hrsg) Social and family issues in shift work and non standard working hours. Springer, Heidelberg, S 39-56

Arlinghaus, A, Gärtner, J (2020) Unfallrisiko verschiedener Schichtsysteme - Simulation mit dem XIMES-Risikorechner. Sozialpolitik.ch 3/2020 - Forum 3.1

Baer K, Ernst G, Nachreiner F, Schay T (1981) Psychologische Ansätze zur Analyse verschiedener Arbeitszeitsysteme. Z Arb Wiss 35:136-141

Blasche G, Bauböck VM, Haluza D (2017) Work-related self-assessed fatigue and recovery among nurses. Int Arch Occup Environ Health 90(2):197-205

BMAS (Hrsg) (2020) Sicherheit und Gesundheit bei der Arbeit - Berichtsjahr 2019. BAuA, Dortmund. https://doi.org/10.21934/baua: bericht 20201215 
Bockelmann M, Arlinghaus A, Nachreiner F (2016) Disability for service in public transport operations: risk factors and interventions. In: Demel B, Stock P, Bruder R, Schlick C (Hrsg) Advances in ergonomic design of systems, products and processes. Springer Vieweg, Berlin, Heidelberg, S 137-147

Bonin H, Eichhorst W, Krause-Pilatus A, Zinne U, Jungnickel V (2021) Wirksamkeitsanalyse der Corona-Maßnahmen - Kurzexpertise. Forschungsbericht 573 des BMAS. BMAS, Bonn

COVID-19-Arbeitszeitverordnung Verordnung zu Abweichungen vom Arbeitszeitgesetz infolge der COVID-19-Epidemie, (COVID-19Arbeitszeitverordnung - COVID-19-ArbZV) vom 07.04.2020, BAnz AT 09.04.2020 V2

Ernst G, Baer K, Diekmann A, Nachreiner F (1983) Zur Interferenz unterschiedlicher Arbeitszeitregelungen mit der Nutzbarkeit der Freizeit. Psychol Prax 27:111-118

Fischer D, Lombardi DA, Folkard S, Willetts J, Christiani DC (2017) Updating the "risk index": a systematic review and meta-analysis of occupational injuries and work schedule characteristics. Chronobiol Int. https://doi.org/10.1080/07420528.2017.1367305

Giebel O, Schomann C, Nachreiner F (2009) Zur Prognose gesundheitlicher Beeinträchtigungen anhand von Merkmalen des Arbeitszeitsystems - Kreuzvalidierung eines Risikoindexes. In: Gesellschaft für Arbeitswissenschaft (Hrsg) Arbeit, Beschäftigungsfähigkeit und Produktivität im 21. Jahrhundert. GfA-Press, Dortmund, S 393-396

Graf O (1922) Über lohnendste Arbeitspausen bei geistiger Arbeit. Psychol Arb 7:548-611

Hacker W, Richter P (1980) Psychische Fehlbeanspruchung: Psychische Ermüdung, Monotonie, Sättigung und Streß. Spezielle Arbeits- und Ingenieurpsychologie in Einzeldarstellungen. Lehrtext 2. VEB DVW, Berlin

Haenecke K, Tiedemann S, Nachreiner F, Grzech-Sukalo H (1998) Accident risk as a function of hour at work and time of day as determined from accident data and exposure models for the German working population. Scand J Work Environ Health 24:43-48

Härmä M, Koskinen A, Sallinen M, Kubo T, Ropponen A, Lombardi DA (2020) Characteristics of working hours and the risk of occupational injuries among hospital employees: a case-crossover study. Scand J Work Environ Health. https://doi.org/10. 5271/sjweh.3905

MAGS NRW (Ministerium für Arbeit, Gesundheit und Soziales des Landes Nordrhein-Westfalen) (Hrsg) (2019) Überwachungsaktion. „Faire Arbeit in der Fleischindustrie“. Abschlussbericht. MAGS, Düsseldorf

Nachreiner F (2017a) Dauer der Arbeitszeit. In: Romahn R (Hrsg) Arbeitszeit gestalten: Wissenschaftliche Erkenntnisse für die Praxis. Metropolis, Marburg, S 105-120

Nachreiner F (2017b) Lage der Arbeitszeit - Schichtarbeit und andere unübliche Arbeitszeiten. In: Romahn R (Hrsg) Arbeitszeit gestalten: Wissenschaftliche Erkenntnisse für die Praxis. Metropolis, Marburg, S 121-146

Nachreiner F, Arlinghaus A, Greubel J (2019) Variabilität der Arbeitszeit und Unfallrisiko. Z Arbeitswissenschaft 73:369-379. https:// doi.org/10.1007/s41449-019-00172-z
Nachreiner F (2020) Arbeitsschutz nach Art der BRD - am Beispiel der Offshore-Arbeitszeitverordnung, ihrer Evaluation und Umsetzung. In: Trimpop R, Fischbach A, Seliger I, Lynnyk A, Kleineidam N, Große-Jäger A (Hrsg) 21. Workshop Psychologie der Arbeitssicherheit und Gesundheit - Gewalt in der Arbeit verhüten und die Zukunft gesundheitsförderlich gestalten! Asanger, Kröning, S 183-186

Nachreiner F, Wirtz A, Dittmar O, Schomann C, Bockelmann M (2010) Study on health and safety aspects of working time. European Commission DG for Employment, Social Affairs and Equal Opportunities Study to support an Impact Assessment on further action at European level regarding Directive 2003/88/EC and the evolution of working time organisation. Annex 1. Brussels, 21. Dez. 2010

Offshore-Arbeitszeitverordnung Verordnung über die Arbeitszeit bei Offshore-Tätigkeiten (Offshore-Arbeitszeitverordnung - Offshore-ArbZV) vom 5. Juli 2013 (BGB1. I S. 2228)

Regulativ über die Beschäftigung jugendlicher Arbeiter in Fabriken vom 9. März 1839

Rinke A (2020) Lehren aus Wuhan: „Ärzte und medizinisches Personal sollten nur sechs Stunden arbeiten“. Interview mit Eckhard Nagel, Präsident des Chinesisch-Deutschen Freundschaftskrankenhauses in Wuhan. Riff Reporter. https://www.riffreporter.de/coronavirus/corona-wuhan-exit-maskenpflicht-krankenhaus-interviewnagel/. Zugegriffen: 20. Nov. 2020

Rivers WHR, Kraepelin E (1896) Über Ermüdung und Erholung. Psychol Stud 1:627-678

Rohmert W, Rutenfranz J (Hrsg) (1983) Praktische Arbeitsphysiologie, 3. Aufl. Thieme, Stuttgart

Rutenfranz J, Knauth P, Nachreiner F (1993) Arbeitszeitgestaltung. In: Schmidtke H (Hrsg) Ergonomie. Hanser, München, S 574-599

SARS-CoV-2-Arbeitsschutzregel (Fassung vom 10. Aug. 2020) Bonn: BMAS

SARS-CoV-2-Arbeitsschutzstandard vom 16.04.2020, Bonn: BMAS

Schmidtke H (1965) Die Ermüdung. Theorien-Symptome-Messversuche. Huber, Bern

Schmidtke H (Hrsg) (1993) Ergonomie. Hanser, München

Schneider H (1911) Gefahren der Arbeit in der chemischen Industrie. Dörnke \& Mey, Hannover

Schomann C, Giebel O, Nachreiner F (2006) Development and preliminary validation of an index for indicating risks of the design of working hours to health and well-being. Chronobiol Int 23(6): 1401-1408

Teiß1 L (1928) Ermüdung und Arbeitszeit als Unfallveranlassung. Reichsarbeitsblatt 8(35):231-237

Vernon HM (1921) Industrial fatigue and efficiency. Routledge, London

Wedderburn AAI (1981) Is there a pattern in the value of time off work? In: Reinberg A, Vieux N, Andlauer P (Hrsg) Night and shift work. Biological and social patterns. Pergamon, Oxford, S 495-504

Wirtz A, Nachreiner F (2012) Effects of lifetime exposure to shift work on fitness for duty in police officers. Chronobiol Int 29(5):595-600 